\title{
Le Département, un acteur clef de la politique de l'eau
}

The "Département", a key-actor in French water policy

\section{Agnès Grandgirard, Rémi Barbier et Marie Tsanga Tabi}

\section{(2) OpenEdition}

\section{Édition électronique}

URL : http://journals.openedition.org/economierurale/278

DOI : 10.4000/economierurale. 278

ISSN : 2105-2581

\section{Éditeur}

Société Française d'Économie Rurale (SFER)

\section{Édition imprimée}

Date de publication : 15 février 2009

Pagination : 22-33

ISSN : 0013-0559

\section{Référence électronique}

Agnès Grandgirard, Rémi Barbier et Marie Tsanga Tabi, « Le Département, un acteur clef de la politique de l'eau », Économie rurale [En ligne], 309 | Janvier-février 2009, mis en ligne le 01 janvier 2011, consulté le 01 mai 2019. URL : http://journals.openedition.org/economierurale/278 ; DOI : 10.4000/ economierurale. 278 


\section{Le Département, un acteur clef de la politique de l'eau}

Agnès GRANDGIRARD, Rémi BARBIER, Marie TSANGA TABI • Unité mixte de recherche Cemagref-École nationale du génie de l'eau et de l'environnement (Engees) en gestion des services publics, Strasbourg

$D$ ans le sillage des révolutions agricole, industrielle et urbaine qui sont la marque des deux derniers siècles, la gestion de l'eau en France a été développée selon une logique essentiellement sectorielle : chaque usage eau potable, eau agricole, eau industrielle, eau de navigation - était organisé de manière relativement autonome, avec ses acteurs, enjeux et règles spécifiques. En particulier, l'alimentation en eau potable fut prise en charge par les communes et, progressivement, par les intercommunalités (Pezon et Petitet, 2004). La loi sur l'eau de 1964, avec la création des agences et des comités de bassin, fut une première tentative pour parvenir à une approche plus intégrée de la gestion des ressources ${ }^{1}$, dans le respect d'un principe de subsidiarité conférant une réelle autonomie aux usagers de chaque bassin hydrographique ${ }^{2}$. Mais si d'importants progrès ont été accomplis depuis, notamment en matière d'épuration des eaux urbaines et industrielles, la situation demeure globalement préoccupante. Les données de l'Institut français de l'environnement (Ifen) attestent ainsi d'une large contamination des eaux superficielles ou souterraines par les nitrates

1. S'il n'existe pas sur le plan théorique d'approche unifiée de la notion de gestion intégrée, CalvoMendieta (2005) considère dans le cas de l'eau « que la notion de gestion intégrée appelle une certaine transversalité dans la définition et l'application des politiques publiques sectorielles» qui y sont liées.

2. On reprend ici la caractérisation proposée par Barraqué (1997) : «C'est quand des communautés locales d'usagers ont le pouvoir de se répartir la ressource qu'on a des institutions subsidiaires ». Les agences de l'eau représentent selon lui une « subsidiarité en mode mineur », dans la mesure où elles ne disposent ni de la maîtrise d'ouvrage ni du pouvoir de police. et les pesticides. Par ailleurs, de manière sporadique ou plus structurelle, des déséquilibres entre l'offre et la demande apparaissent sur de nombreux territoires. Dans ce contexte, l'inadéquation du système actuel de gestion de l'eau fait globalement consensus parmi les acteurs : ce système souffre notamment d'une trop grande complexité et d'un émiettement des responsabilités.

À cet égard, dans le prolongement de la logique mise en place par la loi de 1964, une solution semble disposer de nombreux atouts : la gestion de l'eau « en bien commun » dans les « territoires naturels » que sont les bassins versants, le « petit cycle de l'eau $»^{3}$ continuant à relever de l'échelon communal ou intercommunal. L'introduction par la loi sur l'eau de 1992 des schémas directeurs et des schémas d'aménagement des eaux (SDAGE/SAGE), destinés à fixer pour chaque bassin ou sous-bassin les " orientations fondamentales d'une gestion équilibrée de la ressource en eau », marque une étape supplémentaire importante dans ce processus d'intégration des politiques sectorielles. Loin d'être un cas isolé, cette évolution témoigne plus généralement de la montée en puissance du «paradigme de la gestion intégrée » dans la doctrine et les pratiques de gestion des ressources naturelles.

Pour autant, le débat sur l'échelle et la nature de la coordination à instaurer entre les multiples acteurs de l'eau ${ }^{4}$ est loin d'être

3. Cycle de l'eau destinée à la consommation humaine : prélèvement en milieu naturel, production et distribution de l'eau potable; collecte, transport et traitement des eaux usées.

4. Nous laissons ici de côté la question spécifique de l'administration de l'eau, qui fait elle-même l'objet d'une « rénovation à poursuivre », pour reprendre les termes de Keller (2007). 
tranché. Dans une mise en perspective internationale, Barrow (1998) a ainsi souligné le fait que la gouvernance de bassin n'avait pas toujours, loin s'en faut, tenu toutes ses promesses, soit que les institutions de bassin aient été trop faibles pour peser sur les décisions de gestion effective, soit qu'elles se soient révélées à l'inverse minées par la bureaucratie et/ou la corruption. Blomquist et Schlager (2005) vont même plus loin dans cette idée en affirmant qu'une institution de bassin ne peut à elle seule résoudre les problèmes de choix collectif que soulève la gestion de l'eau. De leur côté, Mermet et Treyer (2001) conviennent certes que «les limites naturelles de la ressource doivent rester une pierre angulaire de la définition des limites de l'unité territoriale de gestion", notamment parce que le bassin versant constitue une bonne échelle de description des fonctionnements des flux d'eau. Mais ils ajoutent que le choix d'une base territoriale adéquate doit également tenir compte de sa capacité à favoriser l'émergence d'une « communauté de gestion de l'eau ». Cela conduit les auteurs à envisager un double amendement à la logique du bassin versant, pour tenir compte des territoires reliés par les systèmes techniques de mobilisation et de distribution de l'eau et des multiples acteurs susceptibles de structurer à moyen/long termes l'évolution de la demande en eau.

Le but de l'article est de prolonger le débat autour du scénario de la gestion intégrée de bassin versant, en montrant ce qui se joue actuellement en France autour du couple département/Conseil général. Le territoire départemental ${ }^{5}$ et son institution politique apparaissent en effet particulièrement dynamiques dans la recomposition actuelle du paysage de la gestion de l'eau. Plus précisément, à travers la diversité de leurs

\footnotetext{
5. Dans cet article, nous faisons le choix de faire la distinction entre le département en tant que territoire que nous écrirons avec un d minuscule et le Département en tant qu'acteur local, c'est-à-dire le Conseil général que nous écrirons avec un $\mathrm{D}$ majuscule.
}

interventions et grâce à leur implication transversale en matière de connaissances et d'évaluation, ils paraissent à même d'établir et de faire vivre un lien entre la protection de la ressource, la lutte contre les inondations, l'appui aux investissements des collectivités et l'accès à l'eau pour les plus démunis. Dans la première partie de cet article, nous revenons sur les atouts de ce « modèle départemental ». Nous détaillons ensuite, dans une deuxième partie, l'action des Conseils généraux dans le domaine de l'eau, et présentons les termes du débat sur la notion de « chef de file » des politiques de l'eau. Enfin, dans une troisième partie, nous illustrons les développements précédents à partir de l'action du Conseil général du Bas-Rhin, un Département pionnier pour ses politiques d'appui aux collectivités et de gestion de la ressource, ainsi que pour son engagement dans une démarche d'évaluation à travers la mise en place d'un Observatoire départemental de l'eau.

\section{Les atouts du modèle départemental}

Dans une perspective générale d'action publique territoriale, nous analyserons dans cette partie les atouts du territoire départemental, puis ceux de son acteur clef, le Conseil général, dont l'influence s'est accrue ces dernières années, en dépit des critiques anciennes concernant son caractère obsolète et à rebours des débats qui avaient précédé la dernière vague de décentralisation.

\section{Le département, territoire clef de l'action publique}

Créés principalement selon des critères géographiques (leurs frontières sont souvent constituées par des rivières ou des montagnes), les départements ont connu différentes phases et ont rempli différents rôles au cours de l'histoire. Le géographe Béhar a souligné récemment l'originalité de cette collectivité : « Dans un contexte où chaque institution - existante ou émergente - cherche à démontrer la pertinence de son territoire en regard des réalités socio-économiques, le Département constitue 
une exception notable: il ne structure pas un "territoire" au sens anthropologique du terme, il dessine un "périmètre" de déploiement de l'action publique, au service de la gestion des territoires » (Béhar, 2002). Cet espace fonctionnel apparaît par ailleurs comme un maillon essentiel dans l'actuel équilibre des pouvoirs locaux, dans la mesure où, selon Cadiou et Maubert (2005), il « s'impose comme un cadre intermédiaire de structuration du pouvoir territorial ». Que faut-il entendre par là ? D'abord, le département constitue un échelon intermédiaire du point de vue administratif, puisqu'il correspond à la fois à un échelon de la décentralisation entre les communes et les régions, mais aussi à un échelon d'organisation des services de l'État. Ensuite, au niveau politique, il est le territoire où se forment des réseaux et des positions de pouvoir qui influent sur d'autres institutions publiques (tels le Sénat, le Conseil régional...). Enfin, au niveau territorial proprement dit, il est constitué par la juxtaposition de villes et de communes rurales, ce qui le met en position a priori favorable pour tenter de concilier les intérêts et contraintes de ces deux types d'espace.

Cette place importante dans le cadre institutionnel français trouve une traduction concrète au niveau de l'organisation de nombreux services publics en réseaux, ou services publics essentiels. Poupeau montre ainsi que, malgré les nombreuses critiques dont il a été l'objet, le département est un échelon prépondérant et dynamique dans le domaine de l'électricité. «Le redéploiement d'EDF met donc en exergue le dynamisme du système départemental français, qui a su tirer profit des évolutions récentes pour renforcer son poids sur la scène locale. [...] Le département continue toujours, pour le moment, d'être le point de référence institutionnel local et ce malgré l'amorce récente par EDF d'une régionalisation de son activité de distribution. » (Poupeau, 2001). Dans le même article, l'auteur déclare que ce dynamisme départemental n'est pas propre à l'électricité mais peut aussi s'observer dans d'autres services publics à caractère industriel et commercial, tels les déchets et l'eau. Rappelons effectivement à cet égard que le législateur a instauré en 1992 une planification départementale de la gestion des déchets ménagers et assimilés. D'abord confié au préfet et à ses services, cet exercice de planification est placé depuis 2005 sous la responsabilité du président du Conseil général. Par ailleurs, dans son analyse des territoires de l'eau, Ghiotti (2006) estime que "l'échelle départementale possède des capacités techniques, financières et administratives qui en font un acteur incontournable (DDAF, DDE, DDASS, services du Conseil général...) ». Ce caractère incontournable pourrait d'ailleurs être conforté par l'apparition de nouvelles contraintes - normes relatives à l'eau potable, sécurisation de l'approvisionnement en eau potable, renouvellement des canalisations qui tendent à favoriser les regroupements de collectivités à l'échelle départementale, où une forme de mutualisation des efforts et des risques peut être mise en œuvre.

De même que le département s'affirme comme territoire structurant pour les prestations marchandes des services publics essentiels, le modèle d'organisation des «prestations de cohésion sociale $»^{6}$ qui s'impose progressivement dans le paysage français s'affranchit du cadre local de référence et investit le département. Ainsi, lorsqu' au début des années 1990 il s'est agi, face à la montée sans précédent des impayés d'eau, de concevoir le maintien à l'eau des populations défavorisées ${ }^{7}, c^{\prime}$ est d'emblée à l'échelle du département qu'a été conçue la gestion de la

6. Nous empruntons à Gadrey (1998) le terme «prestations sociales» pour rendre compte de la composante sociale des missions de service public. 7. La première charte «Solidarité Eau » a été signée en novembre 1996 entre l'État, les opérateurs privés des réseaux d'eau représentés par le Syndicat professionnel des entreprises de service d'eau et d'assainissement (le SPDE) ; et les collectivités locales représentées par l'Association des Maires de France (l'AMF) et la Fédération nationale des collectivités concédantes et régies (FNCCR). 
cohésion sociale dans le monde pourtant très «local » de l'eau, dans le prolongement de l'expérience antérieure des fonds départementaux énergie, logement et téléphone. Le département est apparu comme le territoire d'action pertinent de la lutte contre l'exclusion aux services publics essentiels, car même si le problème avait pu être résolu à l'échelle de chaque commune, on en serait venu à un moment ou à un autre à se focaliser sur les disparités et les écarts entre collectivités locales. La question de la cohésion sociale a pris alors une dimension supra-locale, avec le principe d'une territorialisation plus large de l'action de solidarité et de l'idéal de justice recherché.

\section{Le Département, un acteur qui résiste à la critique}

Ce territoire structurant est par ailleurs doté d'un acteur, le Conseil Général, dont l'évolution récente conforte notre analyse des atouts du modèle départemental. Évoquant le rôle des Départements un peu plus de dix ans après la première vague de décentralisation, Le Galès notait ainsi que ceux-ci étaient " en train de devenir la super-collectivité d'encadrement et de contrôle des communes rurales, tout en négociant en force au sein des régions » (Le Galès, 1995). Le Département jouerait ainsi le rôle de «nouvel intercesseur territorial », selon l'expression de Béhar (op. cit.). Le Galès ajoutait : «Les Départements ont aussi renforcé leurs ressources, leurs capacités d'actions et d'innovation, et sans doute leur poids politique dans l'ensemble du système politique français ». Quelques années plus tard, lors des débats ayant précédé la dernière vague de décentralisation, l'institution départementale a fait l'objet de multiples critiques. De nombreux auteurs et acteurs soulevèrent en premier lieu le problème de l'emboîtement des structures territoriales. Coincé entre la Région, érigée au rang de «nouveau paradigme de l'organisation territoriale » (Piron, 2006), et la montée en puissance des structures intercommunales « de projet » dotées d'une fiscalité propre ${ }^{8}$, le Département était accusé d'être à la fois trop éloigné du terrain et trop petit pour l'élaboration d'une vision globale et stratégique. L'autre critique majeure concernait l'illisibilité du partage des compétences résultant du trop grand nombre de niveaux de collectivités : «La complexité de la répartition des compétences entre l'État et les collectivités territoriales tient, dans une large mesure, à l'existence de plusieurs niveaux d'administration imbriqués sur un même territoire, conjuguée à la prohibition de toute tutelle d'une collectivité sur une autre» (Fonrojet, 2004). Tirant un bilan critique de la décentralisation, Regourd (2004) estimait alors qu' « il n'est pas certain que les Départements, et leurs découpages cantonaux, correspondent à une réelle exigence de la modernité administrative, mais il s'agit là d'une question qui relève du tabou politique ». Et en effet, tabou politique ou réelle pertinence en termes d'action publique, ces « espaces politiques de pouvoir fortement institutionnalisés » (Cadiou et Maubert, 2005) que sont les Départements ont été globalement renforcés par la dernière vague de décentralisation.

Dans ce tableau d'ensemble très favorable, l'évolution de la politique de l'eau est par contre plus ambiguë. D'un côté, alors qu'à la naissance des politiques publiques de maintien à l'eau des publics défavorisés, en 1996, plusieurs acteurs départementaux avaient pu jouer le rôle de chef de file des dispositifs de solidarité, en particulier les directions départementales des affaires sanitaires et sociales et les préfectures, la loi relative aux libertés et responsabilités locales de $2004^{9}$ a transféré la gestion du dispositif aux Conseils généraux. Mais d'un autre côté, en retrait par rapport aux appels en faveur d'une « départementalisation

8. Il s'agit des communautés de communes, d'agglomération et urbaines.

9. Article 65 de la loi n 2004-809 du 13 août 2004 relative aux libertés et responsabilités locales. Voir aussi le décret d'application du 2 mars 2005 relatif au fonds de solidarité pour le logement. 
de la gestion de l'eau » (Launay, 2003), la loi sur l'eau et les milieux aquatiques de décembre 2006 n'a pas été très favorable aux Départements. Elle les autorise certes à mettre à la disposition des communes ou intercommunalités qui ne bénéficient pas de moyens suffisants un nouveau service d'assistance technique pour la protection de la ressource en $\mathrm{eau}^{10}$, mais elle a refusé par ailleurs de transférer aux Départements une partie de la fiscalité assise sur l'eau, confirmant ainsi, après la suppression du Fonds national de développement des adductions d'eau (FNDAE) et sa « reprise » par les Agences, le rôle pivot de ces dernières en termes de financement de la politique de l'eau. Il n'en demeure pas moins que le bilan global des dernières évolutions semble plutôt favorable aux Départements. Une interprétation possible serait que le « département-territoire » a été sauvé par le «Département-acteur politique structurant ». On peut enfin faire l'hypothèse que le futur paysage de la gestion de l'eau sera modelé autant par ce recadrage législatif que par le jeu propre des acteurs, lesquels semblent bien agir en fonction de cette « loi » énoncée par Muller qui veut que «quelle que soit sa compétence réglementaire, une collectivité (département ou région) se saisira d'un problème à partir du moment où il émerge au sein de l'agenda public local » (Muller, 1990).

\section{Les Conseils généraux et l'eau Des "politiques volontaristes diversifiées ॥"}

L'arrière-plan institutionnel étant précisé, nous allons désormais nous intéresser plus spécifiquement aux politiques de l'eau des Conseils généraux (CG). Nous commencerons

10. Article 73 de la loi ${ }^{\circ}$ 2006-1772 du 30 décembre 2006 relative à l'eau et aux milieux aquatiques. Le décret d'application du 30/12/2007 précise les bénéficiaires de cette assistance (les communes rurales les plus pauvres et les petites intercommunalités). Il prévoit également la publication d'un barème de rémunération par chaque Conseil Général concerné. 11. Nous empruntons cette qualification à l'étude ADF - CFE (2003). par rappeler la nature des compétences exercées, avant de faire état de la revendication des Départements à jouer un rôle de chef de file de la politique de l'eau, de présenter les relais de cette revendication et les arguments à l'appui de celle-ci.

\section{Un large éventail d'interventions}

Dans le domaine de l'eau, les Départements interviennent tout d'abord et principalement au titre de leur mission d'assistance à l'équipement des communes. Ils participent ainsi au financement et au suivi de projets ayant trait à la préservation de la qualité des eaux brutes et à l'alimentation en eau potable. En 2004, les 72 Départements ayant répondu à l'enquête du Cercle français de l'eau réalisée pour le compte de l'Assemblée des départements de France (ADF - CFE, 2006) ont ainsi consacré 561 millions d'euros à l'eau et à l'assainissement. Les autres compétences obligatoires des Départements sont les suivantes :

- la participation au schéma directeur d'aménagement et de gestion des eaux lorsqu'il existe ;

- l'aménagement, l'entretien et l'exploitation des cours d'eau, canaux, lacs et plans d'eau domaniaux qui leur sont transférés ;

- le pilotage du maintien de l'accès à l'eau des plus démunis, via la gestion du Fonds solidarité logement (FSL).

Mais les Départements peuvent s'investir au-delà de ce premier ensemble de compétences. À la demande des Agences de l'eau et du ministère de la Santé, la plupart des Départements ont ainsi mis en place depuis les années 1970 un service d'assistance technique aux exploitants de stations d'épuration (SATESE), devenu officiellement compétence optionnelle des départements en $1992^{12}$. Ce service est financé conjointement par le Conseil général et l'Agence de l'Eau. Initialement, le rôle du SATESE se

12. Sur les 69 Conseils généraux interrogés par le Cercle français de l'eau, 59 déclarent avoir un SATESE, et 18 un service d'assistance à l'eau potable (ADF - CFE, op. cit.). 
limitait à l'expertise du fonctionnement des stations d'épuration, mais leur champ d'intervention s'est progressivement élargi vers l'assainissement non collectif ${ }^{13}$, et même dans certains cas, comme dans le Bas-Rhin, vers le devenir des sous-produits de l'épuration. Ce faisant, le Conseil général se positionne comme un acteur clef de l'assainissement, secteur qui représente le premier poste de la dépense environnementale du secteur communal en France (Ifen, 2005a). D'autres services d'assistance peuvent être mis en place, destinés notamment à l'entretien des rivières et à l'eau potable.

Outre l'accompagnement financier et l'assistance technique, les Départements interviennent également dans la planification de la gestion de l'eau. En sus de leur implication dans l'élaboration des SAGE, ils peuvent élaborer des schémas départementaux d'eau potable ou d'assainissement (SDAEP). Centrés sur la gestion quantitative et qualitative de l'eau destinée à la consommation humaine, les SDAEP organisent la sécurisation des approvisionnements et planifient les programmes d'amélioration de la distribution ${ }^{14}$. Les CG peuvent enfin se doter d'outils tels que des observatoires afin d'accompagner et de coordonner la gestion de l'eau sur leur territoire. C'est ainsi que près des deux tiers des Départements interrogés par le Cercle français de l'eau déclarent avoir pris en charge la maîtrise d'ouvrage du suivi des ressources en eau (ADF-CFE, 2003). Il nous semble toutefois important de rappeler qu'il existe une grande hétérogénéité de situations et d'implications entre les divers Départements.

13. Il s'agit du contrôle obligatoire des systèmes d'assainissement non collectif et de la possibilité d'assurer leur entretien. Pour une défense et illustration des SATESE, voir le « Dossier d'information ANSATESE / ARSATESE / HORIZON $2015 \mathrm{du}$ 23/09/2007 » de 1'association nationale des SATESE.

14. Voir par exemple pour l'analyse des SDAEP de la Région Poitou-Charentes la note de l'Observatoire Régional de l'Environnement (http://www.eaupoitou-charentes.org/IMG/pdf/SDAEP-2.pdf).

\section{Le Département : chef de file de la politique de l'eau ?}

Certains acteurs ont revendiqué avec force ces dernières années la reconnaissance d'un rôle accru pour le Département. Le député J. Launay et le sénateur G. Miquel, auteurs de rapports sur la politique de l'eau, figurent parmi les plus ardents promoteurs du « rôle pivot du département » et d'une «politique de l'eau orientée vers la départementalisation». Ils sont suivis en cela par une majorité écrasante des Conseils généraux, qui estiment à $93 \%$ que le Département a une véritable légitimité pour devenir «chef de file» des politiques liées à l'eau et à l'assainissement (ADF-CFE, 2003). La référence à la notion de chef de file, aux contours relativement incertains, a surtout comme effet de manifester le souci de concilier le principe d'absence de tutelle d'une collectivité sur une autre avec le constat des difficultés engendrées par l'implication de différents niveaux de collectivités sur un même domaine d'action publique (tourisme, action sociale, eau...). Mais les Départements ne sont pas les seuls à vouloir, en quelque sorte, préempter cette notion. Ils s'inscrivent en concurrence avec les établissements publics territoriaux de bassin $(\text { EPTB })^{15}$, mais surtout avec certaines Régions

15. Concurrence relative dans la mesure où les CG sont très impliqués dans les EPTB ( $c f$. infra). Pour Allanic, directeur de l'EPTB de la Vilaine, «les EPTB souhaitent jouer un rôle moteur dans la coordination du bassin fluvial : dans le domaine de la prévention des inondations; pour les aménagements hydrauliques et le soutien d'étiage; dans le domaine de la restauration des poissons migrateurs ; mais également pour assurer les grands équilibres de la ressource en eau potable. Les nouvelles normes relatives à l'eau potable et les exigences de qualité exprimées par les consommateurs ont pour conséquences un renforcement de la technicité du traitement de l'eau. Les petites structures de production ne sont plus à même d'assurer des tâches à des coûts admissibles. Les regroupements à l'échelle départementale deviendront alors la règle. Cependant, l'organisation générale de la production à un niveau régional et les grandes structures d'alimentation et de transfert d'eau devront être de plus en plus réfléchies à l'échelle des bassins fluviaux » (Allanic, 2003). 
qui, comme la Bretagne et l'Alsace, portent la même revendication.

Quels sont les arguments susceptibles d'étayer la position des Départements ? Nous pouvons en identifier quatre principaux.

Le premier argument est relatif à leur investissement financier important dans la politique de l'eau, notamment par rapport aux Régions. Selon l'Ifen (2005b), les Départements ont investi 1,325 milliard d'euros en 2002 dans l'environnement, les deux tiers de cette dépense étant consacrés à l'eau (soit $883 \mathrm{M€}$ ); de leur côté, les Régions n'y consacrent que $18,2 \%$ de leurs dépenses environnementales, soit 77 millions d'euros par an environ.

Le deuxième argument est que cet engagement financier traduit et accompagne un réel volontarisme politique. Ghiotti (op. cit.) souligne ainsi la volonté politique des Départements de s'impliquer dans le domaine de l'eau (il étudie notamment l'Ardèche, l'Hérault et le Gard). Il avance que cette stratégie se base notamment sur leur capacité à « capter les territoires d'intercession» comme le bassin versant. En témoigne leur engagement dans les contrats de rivière ou les SAGE dans leur phase initiale. À la suite du rapport Launay (2003), Ghiotti souligne également la forte implication des Départements dans la création et le pilotage des EPTB, ces établissements publics chargés à l'origine de missions précises (étiage/inondation) mais qui ont vu s'étendre progressivement leurs domaines d'intervention, au point d'apparaître comme «le bras armé des élus locaux pour aménager et gérer les grands cours d'eau », dans une logique de concurrence avec les Agences de l'eau.

La troisième considération porte sur l'institution départementale qui a la taille et les compétences pour conduire des études, développer de nouveaux outils et de nouvelles méthodologies permettant de renforcer la coordination des acteurs et le pilotage de la politique. On en donnera des illustrations plus loin.
Le quatrième argument, concerne, enfin, le volet social de la politique de l'eau, la responsabilité de chef de file des Conseils généraux qui repose notamment sur le savoir-faire acquis dans le cadre de leurs missions d'action sociale, domaine dont les enjeux ne relèvent en rien des logiques marchandes du service de l'eau. La nécessité d'un acteur chef de file s'imposait d'autant plus que l'expérience pour les acteurs de l'eau était nouvelle, et que l'émiettement du paysage communal, la diversité des acteurs, la superposition des structures et des modes de gestion font très vite basculer la construction du dispositif de solidarité sociale dans la complexité.

Concrètement, quel pourrait être le contenu de la gouvernance exercée par le Département ? Une première indication est fournie par la revendication des Conseils généraux d'avoir, dans le prolongement de ce que nous avons déjà mentionné, « la responsabilité de l'élaboration d'un schéma départemental de l'eau, document d'orientation et de planification de l'action publique, définissant les objectifs quantitatifs et qualitatifs de prélèvement et de rejet (eau potable et assainissement), l'organisation et la gestion des écoulements ainsi que les principaux ouvrages correspondants, en conformité avec les Schéma Directeur d'Aménagement et de Gestion des Eaux » (ADF - CFE, 2003). Il s'agirait donc d'exercer pour l'eau destinée à la consommation humaine un rôle analogue à celui qu'ils vont être amenés à jouer dans le domaine des déchets, avec la responsabilité de l'élaboration des plans départementaux d'élimination des déchets. Un autre volet de cette gouvernance, plus opérationnel, pourrait être la création et/ou le soutien à des syndicats départementaux ayant diverses vocations, de solidarité (péréquation tarifaire, renouvellement des réseaux) ou de maîtrise d'ouvrage (sécurisation des approvisionnements, équipements structurants). Enfin, cette gouvernance aurait un contenu cognitif important, à travers les études, inventaires et autres systèmes d'information et d'indicateurs (cf. infra l'exemple du Bas-Rhin). 
En définitive, on assiste moins à une opposition entre le modèle départemental et celui du bassin versant qu'à une recherche de clarification de leurs complémentarités et de leurs zones de concurrence. Antoine et Roux (2004) proposent à cet égard de faire la distinction entre un territoire de réflexion et de cohérence, le bassin versant, et un territoire d'action, le Département. C'est d'ailleurs la solution en faveur de laquelle plaide le sénateur Miquel : «Entre les communes et le bassin versant, il y a place pour une structure intermédiaire : le Département. Le Département, sur ce sujet, a deux atouts : c'est à la fois une structure de proximité qui a les moyens de conduire une véritable stratégie territoriale de préservation de la ressource. La planification doit rester au niveau du bassin versant et les agences de l'eau sont aujourd'hui des outils irremplaçables dans l'élaboration de stratégies globales mais le département est probablement l'un des meilleurs niveaux opérationnels» (Miquel, 2003). On retrouve l'argument de Béhar d'un acteur fonctionnel/opérationnel agissant en relais ou en soutien auprès de territoires structurés de leur côté selon une logique de souveraineté et d'identité (les collectivités locales) ou de subsidiarité (bassins ou sous-bassins). Et contre les réticences que pourrait susciter ce choix de ne pas supprimer d'étage dans le feuilletage institutionnel de la gestion de l'eau, on peut rappeler avec Kellow (1985) que l'importance n'est pas tant dans le nombre d'acteurs impliqués que dans la qualité des mécanismes de coordination qu'ils mettent en place. Le modèle départemental semble donc pouvoir être un complément intéressant au modèle du bassin versant. Mais comme le décrit la dernière partie, ce rôle de « chef de file opérationnel » n'est pas aisé à construire.

\section{Un éclairage empirique Le cas du Bas-Rhin}

Nous allons désormais illustrer notre propos et mettre en discussion notre conclusion provisoire avec l'exemple du Conseil général du Bas-Rhin $^{16}$. L'ensemble de cette partie s'ap- puie sur une recherche-intervention menée pour le compte du Conseil général du BasRhin à propos de la mise en place d'un Observatoire de l'eau. Dans ce cadre, une trentaine d'acteurs de la gestion de l'eau, élus, techniciens, administratifs ou associatifs, ont été interrogés afin de recueillir leurs attentes visà-vis de l'Observatoire de l'eau.

\section{Un système en voie de polarisation autour du Conseil général ?}

Du point de vue de la gestion de l'eau, le département du Bas-Rhin présente un certain nombre de particularités qu'il convient de préciser. Tout d'abord, sa géographie en fait quasiment un bassin versant naturel. Ensuite, on peut y noter une répartition originale des compétences dans le domaine de l'eau, entre la Région Alsace, responsable des eaux souterraines, et les deux Départements, Bas et Haut Rhin, responsables des eaux superficielles. Enfin, la gestion de l'eau potable et de l'assainissement y est également singulière. En effet, elle est confiée par $80 \%$ des communes au Syndicat départemental de l'eau et de l'assainissement du Bas-Rhin (SDEA). La délégation de service public à des gestionnaires privés est peu répandue, la régie interne représentant la plupart des autres modes de gestion. L'adhésion au SDEA est en forte progression chaque année et les communes isolées sont de plus en plus rares.

Dans ce contexte, le Conseil général du Bas-Rhin (CG67) occupe historiquement une place privilégiée dans l'accompagnement des collectivités dans les domaines de l'eau potable et de l'assainissement, ainsi que dans l'appui à la maîtrise d'ouvrage pour la gestion des cours d'eau. Il est en effet le premier financeur des investissements portés par les communes et les intercommunalités, avec

16. Le volet social de l'action du Conseil général liée à l'accès à l'eau ne sera pas traité ici en raison du caractère récent de son expérience dans ce domaine. Le décret d'application relatif au fonds de solidarité pour le logement qui confie la gestion du dispositif d'accès social à l'eau aux Conseils généraux date en effet de mars 2005. 
16 millions d'euros par an consacrés au financement des réseaux (ce qui le place largement au-dessus de l'investissement moyen des Départements), aux études et travaux de protection, à la renaturation des rivières, à la sécurisation des ressources d'eau potable, ou à la gestion des boues d'épuration. Par ailleurs, il revendique « un rôle fédérateur de la politique de l'eau», mettant en avant son «statut de seul acteur public agissant sur l'ensemble du cycle de l'eau », de la protection de la ressource à la gestion des boues d'épuration en passant par le co-financement des équipements. Enfin, le Conseil général souligne qu' « au-delà des prescriptions réglementaires qui s'imposent à tous », une de ses ambitions consiste bien à «mettre le plus possible en cohérence les politiques de l'eau et d'aménagement du territoire $\gg{ }^{17}$. On le voit, l'ambition d'intégration est à double détente : il s'agit d'abord d'intégrer les diverses composantes de la gestion des eaux destinées à la consommation humaine (de la protection de la ressource à l'épandage des boues), mais aussi de dépasser ce cadre qui demeure sectoriel pour aller vers une véritable prise en compte de l'ensemble des enjeux territoriaux liés à l'eau.

Pour mener à bien cette perspective, le CG67 a développé de nombreux outils, puisant dans toute la palette des instruments de l'action publique. On citera tout d'abord les outils de planification territoriale négociée, tels les Schéma d'aménagement et de gestion de l'état écologique des cours d'eau ${ }^{18}$, qui ont vocation à évoluer vers des SAGE. On mentionnera également les outils d'in-

17. Source: «Des Hommes et des Territoires. L'eau dans le Bas-Rhin. Réforme de la politique départementale. Porter à connaissance », juin 2004. 18. Les SAGEECE (Schéma d'aménagement et de gestion de l'état écologique des cours d'eau) sont un outil de concertation et de programmation de travaux d'aménagement et d'entretien des rivières. Ils ont été mis en place par le Conseil général du Bas-Rhin avant que les SAGE n'existent. Ils concernent un périmètre plus restreint et se concentrent sur la problématique des cours d'eau. tervention, comme le SATESA ou encore la mission boues, créée en partenariat avec la chambre d'agriculture. Divers types de contrats d'action publique ont également été développés : le contrat-cadre avec l'Agence de l'eau, mais aussi les « contrats de territoire $»$ avec les communes et intercommunalités, contrats qui ont vocation à favoriser l'articulation entre les problématiques de l'aménagement et celles de l'eau. Enfin des outils de connaissance ont été développés, comme les inventaires des réseaux d'eau potable et d'assainissement ${ }^{19}$, et l'Observatoire de l'eau que nous allons présenter plus longuement. C'est en effet un outil intéressant dans la mesure où il souligne et met à l'épreuve une double volonté du CG : mettre en place une logique d'évaluation de ses actions, et fédérer l'ensemble des acteurs de l'eau.

\section{L'Observatoire départemental de l'eau, un outil pour évaluer et fédérer}

Dans le cadre de notre étude, nous avons pu identifier par Internet une dizaine d'observatoires de l'eau, et nous avons mis en évidence une grande hétérogénéité, tant dans les objectifs poursuivis que dans le contenu et le fonctionnement de ces observatoires (Grandgirard et Barbier, 2006). Pour ce qui le concerne, en mettant en place cet observatoire $^{20}$ destiné à un large public, le Département du Bas-Rhin poursuivait un quadruple objectif :

- valoriser les nombreuses données recueillies grâce aux différents outils énumérés précédemment (SATESE, inventaires...) ;

- contribuer à faire émerger une vision globale de l'eau ;

- faciliter l'évaluation de sa politique de l'eau ;

- se mettre en conformité avec la Directive

19. Ils sont originaux dans le sens où cette approche n'avait jamais été menée à un niveau supra-local de cette envergure.

20. Pour plus de développement sur cet outil, nous renvoyons à Grandgirard (2007). 
cadre européenne sur l'eau, qui incite à plus de transparence, notamment en direction des usagers-citoyens auxquels seraient fournis notamment des données techniques expliquée (prix de l'eau, évolution du prix, qualité du service...).

N'étant pas le seul détenteur de données et ambitionnant dès l'origine de profiter de ce projet pour fédérer les acteurs de l'eau, le Conseil général a donc invité ces derniers à s'impliquer dans le projet. C'est ainsi que le comité de pilotage de l'Observatoire comprend des élus locaux en charge des dossiers relatifs à l'eau (Conseillers généraux, maires, présidents de syndicats, en particulier le syndicat départemental), des représentants des diverses institutions de l'eau (Agence de l'Eau Rhin Meuse, DDASS, DIREN, Police de l'Eau), et des représentants des usagers de l'eau : association de consommateurs, fédération de pêche, industriels, chambre d'agriculture... Ce large éventail d'acteurs matérialise la volonté d'ouverture du CG67. Le projet symbolise également le nouveau positionnement que vise le Département dans le système d'acteurs, non plus simple financeur, mais moteur d'initiatives et porteur d'une volonté de pilotage de la gestion de l'eau dans l'ensemble de son cycle.

En janvier 2007, l'Observatoire comptait déjà un certain nombre de produits, parmi lesquels on peut citer : un site Internet (encore peu développé et convivial) ; des synthèses techniques diverses (sur le fonctionnement des stations d'épuration urbaines, sur la production et l'élimination des boues, sur le milieu naturel, sur la gestion de l'eau et de l'assainissement) ; des bilans techniques et financiers par collectivité (alimentation en eau potable/assainissement).

Malgré l'ensemble de ces outils et de ces initiatives, force est de constater qu'il existe des limites au volontarisme intégrateur du CG67. La première tient à la difficulté de concrétiser l'objectif d'observation et d'évaluation, qui constitue pourtant l'infrastructure cognitive clef d'une gestion intégrée de la ressource. L'observation requiert un impor- tant travail préalable de mise en cohérence des données recueillies, afin de garantir notamment leur comparabilité. Cette mise en cohérence est d'autant plus difficile à obtenir que le nombre de producteurs de données est important, et que l'hétérogénéité des protocoles de mesure et des formats de données recueillies est forte. Et en ce qui concerne l'évaluation, les problèmes liés à la construction et à l'usage des indicateurs sont loin d'être tous résolus. En particulier, les indicateurs doivent être maniés avec précaution car la connaissance d'un paramètre de qualité de l'eau ne permet pas forcément une évaluation des actions menées : il faut en effet du temps pour voir les résultats des actions menées, d'autres paramètres agissent et on s'intéresse à des phénomènes complexes. L'évaluation ne peut donc pas se résumer à des chiffres. Ceux-ci ne peuvent que servir de base à une analyse et à une réflexion plus approfondie, ce qui requiert la mise en place d'une réelle politique d'évaluation ; or, celle-ci n'en est encore qu'à ses balbutiements.

L'autre frein auquel se heurte l'action du CG67 est l'implication molle des autres acteurs. Cette situation crée notamment des problèmes de recueil des données, et par ricochet des difficultés de construction des indicateurs, d'évaluation et de gestion de la transversalité nécessaire à la gestion de l'eau dans l'ensemble de son cycle. Or celle-ci est difficilement réalisable sans ce partage de connaissances. Ce qui semble alors manquer est cette autre condition sine qua non d'une véritable gouvernance territoriale, à savoir l'adhésion de l'ensemble des acteurs concernés à un espace de sens commun (Muller et Surel, 1998). Plus généralement, l'absence initiale de communauté départementale des usagers de l'eau constitue sans doute l'un des principaux obstacles à la logique fonctionnelle de l'intervention des Conseils généraux. 


\section{Conclusion}

Dans cet article, nous avons choisi de présenter la place occupée par les Départements dans la gestion des politiques de l'eau. Les évolutions observées ces dernières années permettent de soutenir l'idée de l'émergence d'une politique intégrée de l'eau à l'échelle départementale. On voit en effet se dessiner un système d'acteurs polarisé autour du Conseil général, qui construit et utilise des outils innovants, élargit son action et prend des initiatives qui vont bien au-delà de ses obligations réglementaires. Petit à petit, il se positionne en tant que complément du bassin versant, incontournable dans la mise en place des actions, tirant sa légitimité de sa « proximité » historique d'avec les communes, de l'ancienneté et de l'importance de son action en matière d'aménagement et d'équipements ainsi que de son expertise dans le domaine de l'action sociale.
Ce constat global ne doit pas masquer qu'il existe une grande hétérogénéité dans l'implication des différents Départements, et que leur action demeure financièrement plus ciblée sur l'aide à l'équipement que sur les autres aspects de la gestion de l'eau. Il ne faut pas minorer également le fait que si une certaine intégration de la politique de l'eau destinée à la consommation humaine peut être observée, des contradictions sont encore certainement identifiables avec d'autres politiques départementales, comme le soutien à l'agriculture par le drainage de zones humides par exemple. On le voit, l'évidence d'une gestion intégrée de l'eau aboutie à l'échelle départementale n'est pas encore manifeste mais l'histoire est loin d'être définitivement écrite.

\section{Remerciements}

Les auteurs remercient les deux relecteurs pour leurs remarques et suggestions qui les ont aidés à préciser leur propos.

\section{RÉFÉRENCES BIBLIOGRAPHIQUES}

ADF - CFE (2006). Les Départements et l'eau. Enquête du Cercle Français de l'Eau pour l'Assemblée des Départements de France, avril.

ADF - CFE (2003). Les Départements et l'eau. Enquête du Cercle Français de l'Eau pour l'Assemblée des Départements de France, mars.

Allanic M. (2003). Les Établissements Publics Territoriaux de Bassin, chefs de file de la gestion intégrée ? Intervention à la journée d'étude «Les nouveaux territoires de la gestion de l'eau potable », ENGEES, 23 janvier.

Antoine J., Roux A.-L. (2004). Eau et territoires : vers une gestion intégrée. In Journée d'études : «les territoires de l'eau », Arras, Université d'Artois, p. 157-166.
Barraqué B. (1997). Subsidiarité et politique de l'eau. In Faure A. (Dir.), «Territoires et subsidiarité : l'action publique locale à la lumière d'un principe controversé », Paris, L'Harmattan, p. 165-201.

Barrow (1998). River basin development planning and management: a critical review. In World Development, vol. 26, $\mathrm{n}^{\circ} 1$, p. 171-186.

Béhar D. (2002). Le Département : nouvel intercesseur territorial. In Floquet C. (Dir.), « Pour en finir avec la dé-centralisation », L'Aube.

Blomquist W., Schlager E. (2005). Political pitfals of Integrated Watershed Management. Society \& Natural Resources, vol 18, nº 2, p. 101-117.

Cadiou S., Maubert C. (2005). Au centre des forces locales. Éléments et enjeux d'une réflexion prospective. Rapport $\mathrm{n}^{\circ} 7$. Commissariat général du Plan, 93 p. 
Calvo-Mendieta I. (2005). L'économie des ressources en eau : de l'internalisation des externalités à la gestion intégrée. L'exemple du bassin versant de l'Audomarois. Lille, Université des sciences et technologies, thèse de doctorat en sciences économiques.

Fonrojet S. (2004). L'organisation territoriale : quelle répartition des compétences ? Les Cahiers Français. Décentralisation, Etat et territoires, p. 22-26.

Gadrey J. (1998). Les prestations sociales de La Poste. Rapport pour la Direction de La Poste, $32 \mathrm{p}$.

Ghiotti S. (2006). Les territoires de l'eau et la décentralisation. La gouvernance de bassin versant ou les limites d'une évidence. Développement Durable \& Territoires, $28 \mathrm{p}$.

Grandgirard A. (2007). De la gestion intégrée comme doctrine à l'intégration comme défi de gestion. Paris, Ecole Nationale Supérieure des Mines de Paris, thèse en sciences de gestion, $285 \mathrm{p}$.

Grandgirard A., Barbier R. (2006). Les observatoires de l'eau, des outils au service de l'évaluation? Développement Durable et Territoires, dossier $n^{\circ} 8$.

IFEN (2005a). Le rôle majeur de l'intercommunalité dans la gestion publique de l'environnement. Les données de l'environnement, $\mathrm{n}^{\circ} 100,4 \mathrm{p}$.

IFEN (2005b). Les dépenses d'environnement des départements et des régions (1996-2002). n 2, $26 \mathrm{p}$.

Keller (2007). Politique de l'eau : la France au milieu du gué. Rapport à la Commission des finances, du contrôle budgétaire et des comptes économiques de la nation. Paris, Sénat, 58 p.

Kellow A.-J. (1985). Managing an ecological ecosystem 2: the politics of administration. Australian Quaterly, vol. 57, $\mathrm{n}^{\circ} 1 \& 2$, p. 107-27.
Launay J. (2003). La gestion de l'eau sur le territoire, Assemblée nationale, Délégation à l'aménagement et au développement durable du territoire. Rapport d'information $\mathrm{n}^{\circ} 1170$, novembre, $93 \mathrm{p}$.

Le Galès P. (1995). Du gouvernement des villes à la gouvernance urbaine. Revue française de Science Politique, vol. 45, p. 57-95.

Mermet L., Treyer S. (2001). Quelle unité territoriale pour la gestion de l'eau ? Annales des Mines, Responsabilité et Environnement, $\mathrm{n}^{\circ} 23$, p. 67-79

Miquel G. (2003). La qualité de l'eau et de l'assainissement en France. Rapport de l'OPECST, n ${ }^{\circ} 215$.

Muller P., Surel Y. (1998). L'analyse des politiques publiques. Paris, Montchrestien, $156 \mathrm{p}$.

Muller P. (1990). Les politiques publiques entre secteurs et territoires. Politiques et Management Public, vol. 8, $\mathrm{n}^{\circ} 3$.

Pezon C., Petitet S. (2004). L'intercommunalité en France de 1890 à 1999, la distribution d'eau potable en question. In Actes de la journée d'études « Les territoires de l'eau », Arras, Université d'Artois, 26 mars.

Piron M. (2006). Rapport d'information sur l'équilibre territorial des pouvoirs. Paris, Assemblée Nationale, Rapport $\mathrm{n}^{\circ} 2881$, $354 \mathrm{p}$.

Poupeau F.-M. (2001). Libéralisation du service public et action publique locale. Le département dans la recomposition du système de distribution électrique français. Sociologie du travail, $\mathrm{n}^{\circ} 43$, p. 179-195.

Regourd S. (2004). L'organisation territoriale issue de la décentralisation de 1982 : un bilan critique. Les Cahiers Français. Décentralisation, Etat et territoires, p. 3-7. 